\title{
Hysteresis in Hand-Eye Coordination
}

\author{
John G. Holden \\ Arizona State University \\ Tempe, AZ 85287
}

\begin{abstract}
1
A perceptual displacement paradigm is used to explore the dynamic properties of hand-eye coordination. Participants used a computer mouse to trace the outline of a circle that was displayed on a computer monitor. The experimental apparatus introduced a series of rotational displacements to the participant's normal hand-eye coordination. These displacements were similar to the kinds of coordination displacements that are associated with performing endoscopic surgery. The goal of the investigation was to test whether the coordination system displayed behavior similar to that associated with simple nonlinear dynamic systems, such as hysteresis and multistability. The observed hysteresis pattern parallels in quality the hysteresis behavior of rather simple nonlinear dynamic systems. Hysteresis underscores the role of the contextual constraints in both an understanding and control of behavior. It also motivates dynamic systems theory as a general framework for understanding coordination.
\end{abstract}

\section{Introduction}

Endoscopic surgery is a generic term for the surgical technique that uses small incisions and instruments, thus avoiding the large incisions necessary during traditional surgery. During an endoscopic procedure, a compact video camera and special surgical tools are guided into small surgical openings in the patient's body. Due to the small incisions, endoscopic procedures require a shorter recovery period for the patient, and produce much less postoperative pain than an equivalent traditional surgical procedure. However, endoscopic procedures also dramatically complicate the surgeon's task $[9,10,29]$.

Endoscopy is difficult partly because it allows for many possible mappings, or couplings, between the hands and the eyes. The video camera moves independently from the surgical instruments, which repeatedly changes the orientation of how an action looks 1 Copyright 1998 IEEE. Published in the Proceedings of HICS'98, March 22-24, 1998 in Dayton, Ohio. relative to the movement that produces it. In order to remove a gallbladder endoscopically, for example, surgeons insert the video apparatus (endoscope) through the patient's navel. However, the surgical instruments are inserted through small incisions located off to one side of the navel. This off-axis camera-instrument arrangement often results in serious disruptions in hand-eye coordination. In many cases, learning these new coordination relationships is the crux difficulty that surgical residents face as they begin endoscopic training [14]. Careful consideration of factors that facilitate or degrade performance, under conditions that require the use of unusual coordination patterns, may inform the design of an effective training intervention or simulator.

In general, the introduction of remote manipulation (teleoperation) into the workplace requires operators to learn new hand-eye coordination patterns [ $[15$, 18, 21, 29]. Factors such as effecter time lags, and rotations of the hand-eye axis can severely disrupt normal hand-eye coordination [7, 15, 29]. Endoscopic surgery is one example of a domain that requires such skills.

Research aimed at understanding the relationship between perceptual and motor systems has a long history. The works of Helmholtz [16] and Stratton [27, 28] are early examples. Before computers were widely available, researchers manipulated normal coordination using eyepieces, usually prisms, that introduced different types of distortions to visual perception (for reviews, see [19, 36, 37]). Patterns of performance errors motivated hypotheses concerning the structure of hand-eye coordination. More recently, these prismatic adaptation tasks have been modified so they may be implemented on computers (e.g., [6]).

The most general finding is that hand-eye coordination is surprisingly flexible. It adapts to almost any stable rearrangement of coordination $[36,37]$. Preservation of the dynamic coupling between perceptual experience and motor behavior appears to be a key factor for supporting skilled performance and coordination in these tasks $[17,18]$. This article concerns other dynamic patterns, such as hysteresis (history dependent behavior), that may appear in performance domains that require new visuomotor coordination patterns. 
Conventional theories of perceptual-motor coordination tend to minimize the dynamic aspects of performance, and focus instead on relatively static features. Most cases of hand-eye coordination research seek structural invariants that govern the hand-eye system. Bedford [2] suggested, for example, that "It should be possible to discover the rules that operate whenever new [visual-motor] pairs are encountered, such that for every conceivable mapping between the two dimensions, one can predict the behavior that will result." Overall, the goal of many investigations is to discover a lawful relationship between performance error and the characteristics of visual-motor rearrangements ([6]; see [17] for a more detailed discussion of this issue).

It is sometimes suggested that the spatial structure of the system may be based on direction and distance (e.g., $[6,25])$. If so, then the process of learning new coordination patterns may be viewed as a computational process involving the discovery of a suitable transformation from visual coordinate space to motor coordinate space. Bedford [2] suggested that a linear constraint acts on the process of learning new visualmotor relationships. What seems to change during the course of learning is the value of the slope and intercept of a coordination system parameter. Typical learning curves for performance at particular displacements are consistent with this characterization. Error is a negatively accelerated function of amount of displacement exposure [37]. A difference equation, for instance, where the amount of error observed on a particular trial is, on average, a fixed percent of the error observed on the previous trial would display a similar pattern of diminishing error.

The computational, rule-based view suggests that the pattern of errors associated with a perceptual displacement task is fully determined by the amount and type of displacement introduced to the research participant (plus some random variation). The intrinsic dynamics of the coordination are thus relatively static in nature. Behavior is mediated by abstract, fixed representations or functions that transform visual space (i.e., direction and distance) into appropriate motor space representations. The relative difficulty of different coordination displacements are assumed to be independent of each other, which allows that behavioral patterns are due to the structural, rather than dynamic, properties of the coordination system [6]. Such strictly linear systems, however, are a special case of a more general class of dynamic systems. This class includes nonlinear dynamic systems that display a much greater variety of functional relationships. A demonstration of coordination behavior that falls outside of the narrow, linear, "context-free" classification would motivate context sensitive models from the broader class of nonlinear models.

It is possible to skillfully alternate between more than one hand-eye relationship [8, 11, 23]. However, basic research on adaptation to variable prismatic displacement suggests that these coordination patterns are relatively difficult to maintain (e.g., $[38,11$, ]). Nevertheless, endoscopic surgeons do perform effectively, despite the fact that they are often forced to use displacement axes that are relatively variable. The camera moves during surgery, and the displacement of the surgeons normal hand-eye axis changes as a function of these movements.

Several theoretical accounts suggest that coordination of perception and action should be sensitive to task contiguity. Moreover, in actual task domains, such as during endoscopy, perceptual-motor events are contiguous in time. They are events that covary in both space and time $[12,13,22,30,31,33,34]$. The critical insight is the suggestion that coordination between perception and action is an interactive process. Coordination emerges in recurrent flow of sensory-motor patterns that are produced as the trajectory of behavior and behavioral consequences unfold in time [34]. Learning a new hand-eye coordination pattern is an iterative dynamic interplay between perception and action resulting in new stable system behavior. Consequently, we may expect to observe nonlinear, "interactive" patterns in performance. These patterns are analogous to patterns observed in the behavior of simple nonlinear (mathematical) dynamic systemsKi.e., patterns such as multistability and hysteresis, the primary topic of this investigation.

\section{Hysteresis}

Within the conventional theoretical framework, the order in which research participants receive coordination displacements is treated as a nuisance factor. Order effects are not considered theoretically interesting. They are viewed instead as sources of experimental bias or noise. However, randomly ordered displacements preclude the possibility that the coordination system is sensitive to its immediate history.

We may test whether the coordination system is sensitive to, or affected by, its immediate history, which may inform the design of endoscopic or remote manipulation training interventions. Systematic history dependent effects may produce a hysteresis pattern in coordination errors. If so, then this pattern may inform both the applied and theoretical perspectives. For example, if one were interested in explicitly training for the endoscopic environment, it may be helpful to apply an approach that capitalizes on the dynamic characteristics of the coordination system. 
The term hysteresis refers to history dependent behavior. It can arise for systems that sometimes display different outputs to the same system input. Thus it refers to an input-output relation that cannot be described as a single-valued function. This pattern is indicative of multistability [24]. Whether the system displays one or the other output is contingent on the immediate history of outputs. Many physical systems display this property (e.g., forced hard spring, $[1,5,35])$. Figure 1 displays a prototypical example of such a function.

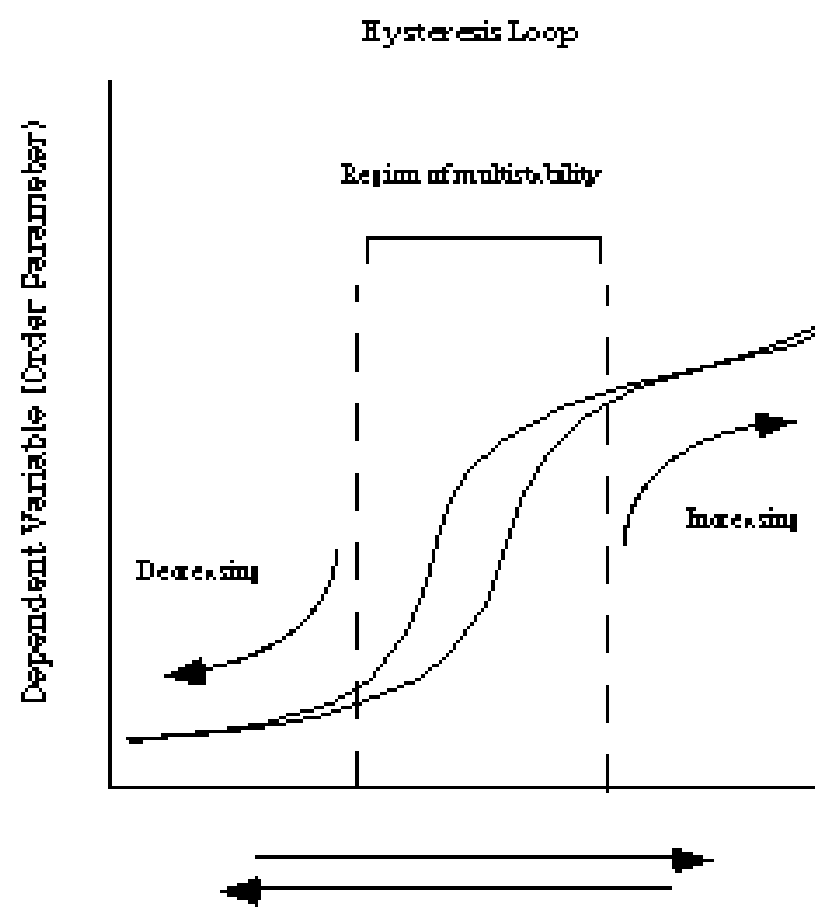

Figure 1. An idealized hysteresis loop. Notice that the system exhibits different behaviors, depending on the order of the control parameter manipulation. This entails the property of multistability, the ability to exhibit different outputs for the same input.

Hysteresis is observed when the system occupies different states, depending on the direction of the control parameter manipulation. The first step is to identify a variable reliably associated with the global behavior of the system. This variable is often referred to as a control parameter. It may be distinguished from the typical use of independent variables, because control parameters affect the relations among system components, rather than one or the other of the components themselves [20, 26]. As the control parameter is systematically changed from, for example, small values to large values, a participant's trial by trial performance is recorded. Next, the manipulation is reversed, and the initially large values of the control parameter are incrementally decreased. Again, trial by trial performance is recorded.

Hysteresis is recognized in many different systems by its distinctive graphical appearance [5]. Hysteresis corroborates the coexistence of multiple stable output states; and the coexistence of multiple stable system states may be regarded as evidence of nonlinearity $[1,20,24,26,32]$.

The present Displacement Experiment tested whether hand-eye coordination displays hysteresis. Participants performed a computer based task that systematically varied the hand-eye axis from 0 degrees (normal coordination) to 180 degrees $($ right $=$ left; up $=$ down) in 20 degree increments. Figure 2 illustrates the resulting cursor movement on a computer monitor for each displacement, from a physical hand movement (of a computer "mouse") directed to the participant's right, approximately parallel to their shoulder axis.

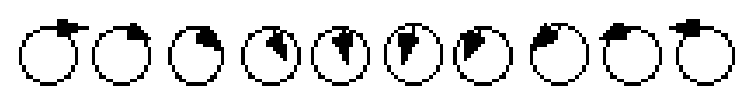

Figure 2. This figure depicts the trajectory of the cursor that results from a physical movement directly to the participants right (see 0 deg.), at the beginning of an experimental trial for each displacement condition.

An ascending displacement manipulation required the participants to perform incrementally increasing displacements (i.e., $0^{\circ}, 20^{\circ}, 40^{\circ}, 60^{\circ}, \ldots 180^{\circ}$ ). The descending manipulation required the participants to perform incrementally decreasing displacements. This experimental manipulation may approximate a control parameter because it changes the relation between vision and the resulting motor output.

\section{Methods}

\section{Participants}

Thirty-two introductory psychology students participated in this experiment for course credit. All were right handed and had normal or corrected to normal vision.

\section{Apparatus and Stimuli}

A color monitor, connected to an IBM compatible computer was used for each experiment. The target stimulus was a flat, red wire frame circle (aprox. $75 \mathrm{~mm}$ in diameter) that appeared in the center of the display screen. A computer mouse was used as an input device to record the participant's hand movements. A series of 
rotational displacements was introduced to the cursor during the course of the experimental trials; they ranged from 0 degrees (no displacement) to 180 degrees (right $=$ left, up = down), in 20 degree increments. A total of ten displacement locations were presented.

\section{Procedure}

Participants used the computer mouse to trace the circumference of the circle that was displayed on the computer monitor. A trial consisted of a single attempt to trace the target circle. Each trial began with the cursor located at the apex of the circle outline. Participants used their right hand to trace the circle in a clockwise direction and they were instructed to keep the mouse upright and in motion during the entire course of the trial. Otherwise they were told to complete their movements at a reasonable pace, comparable to the pace used when completing their first trial, which was always a $0^{\circ}$ displacement. No time limit for completion was imposed on the participants. Occasionally a participant would become completely disoriented during trials that employed displacements larger than $80^{\circ}$. These trials were terminated in cases where the cursor became pinned against an edge of the monitor.

Each participant was presented with two blocks of ten trials. The displacements were presented in order, corresponding to two experimental conditions. The ascending condition began with a $0^{\circ}$ displacement and amount of displacement was progressively increased with each trial in $20^{\circ}$ increments to $180^{\circ}$ (i.e., $0^{\circ}, 20^{\circ}, 40^{\circ}$, $\left.60^{\circ}, 80^{\circ}, 100^{\circ}, 120^{\circ}, 140^{\circ}, 160^{\circ}, 180^{\circ}\right)$. In contrast, the descending condition began with a $0^{\circ}$ practice displacement, then a $180^{\circ}$ displacement was presented, followed by progressively decreasing displacements (i.e., $180^{\circ}, 160^{\circ}, 140^{\circ}, 120^{\circ}, 100^{\circ}, 80^{\circ}, 60^{\circ}, 40^{\circ}, 20^{\circ}$ ).

Each participant completed both order conditions. To control for practice effects across the order manipulations, participants were alternately assigned to begin with either the ascending or descending conditions. This distributed simple practice effects across the displacements in a way that worked against the hypothesized direction of the hysteresis. For example, beginning the task with the ascending trial block fixed the second trial as the $20^{\circ}$ ascending trial, however, starting with the descending trial block established the 12 th trial as the $20^{\circ}$ ascending trial. Thus, on average, the performance that generated the $20^{\circ}$ ascending data cell resulted from seven trials of practice. Alternatively, performance that composed the $20^{\circ}$ descending data cell benefited from 15 trials of practice (i.e., the cell was composed of trials 10 and 20). Consequently, the average number of completed trials that composed the $20^{\circ}$ through $80^{\circ}$ ascending data cells was less than those that formed the corresponding descending cells. Likewise, practice was biased against the $100^{\circ}$ through $180^{\circ}$ descending trials, such that ascending trials corresponding to these displacements had a practice advantage.

\section{Data Acquisition and Analysis}

The experimental software sampled the position of the cursor approximately 17 times per second. The data were collected using the pixel unit of display on computer monitors. One millimeter equaled three and three quarters (3.75) of these units. All data acquisition and analysis was conducted using the pixel units, any metric conversions provided here were completed as a final step to enhance the readability of this document.

The dependent variable for this experiment was root mean squared error, hereafter, simply called the error score. Essentially, this variable represented the average absolute value of the distance from the outline of the target circle during the course of each trial. The radius of the target circle was 100 pixels $(37.5 \mathrm{~mm})$. The raw $\mathrm{X}$ and $\mathrm{Y}$ screen coordinates were transformed according to the following formula: $\left(\left[\left(\mathrm{X}^{2}+\mathrm{Y}^{2}\right)^{1 / 2}-100\right]^{2}\right)^{1 / 2}$. Where $\mathrm{X}$ and $\mathrm{Y}$ refer to the horizontal and vertical locations of the cursor on the video monitor. This transformation subtracts the radius of the target circle from the total distance between the cursor and the center of the circle. If, for example, a participant produced a trace that was always on the target, then it would always be 100 pixels from the center of the circle, and as a result produce an average error of zero pixels.

\section{Results}

The distance from the outline of the target circle for each sampling interval during the duration of a trial was averaged and employed as the dependent measure of performance for each trial.

Five participants found the large displacements $\left(100^{\circ}\right.$ and up) particularly difficult. A total of 7 trials (1\%) were terminated because performance was so poor that the participants were unable to finish tracing the circle. Excluding the data generated by these trials from the analysis did not change the qualitative pattern of the results. Because four of these participants began the task by completing the ascending trials, dropping these trials would bias the practice counterbalancing. Consequently, all data were included in the analysis.

In Figure 3, average error is plotted at each level of displacement for each of the two orders of displacement. The difference between descending and ascending error at each displacement was computed for 
each of the participants. A series of related sample $t$-tests that contrasted ascending and descending performance at each displacement was conducted to test the hysteresis hypothesis. All contrasts employed a $p<.05$ alpha level.

Ascending performance was reliably better for the $20^{\circ}, 40^{\circ}$ and $60^{\circ}$ displacements $(t(31)=2.14, p<$ $.04 ; t(31)=3.11, p<.004 ; t(31)=3.29, p<.003$, respectively). Thus, a hysteresis loop was observed between the $0^{\circ}$ and $80^{\circ}$ displacements. No statistically reliable performance differences were observed for the $80^{\circ}$ and $100^{\circ}$ displacements. Both of these displacements border the $90^{\circ}$ displacement and it is the most difficult displacement to perform using this task. To achieve a desired movement requires that the cursor be moved at a right angle to the intended target location. Behaviorally, this is a very difficult transformation.

\section{Performance Error as a Function of Order and Level of Displacement}

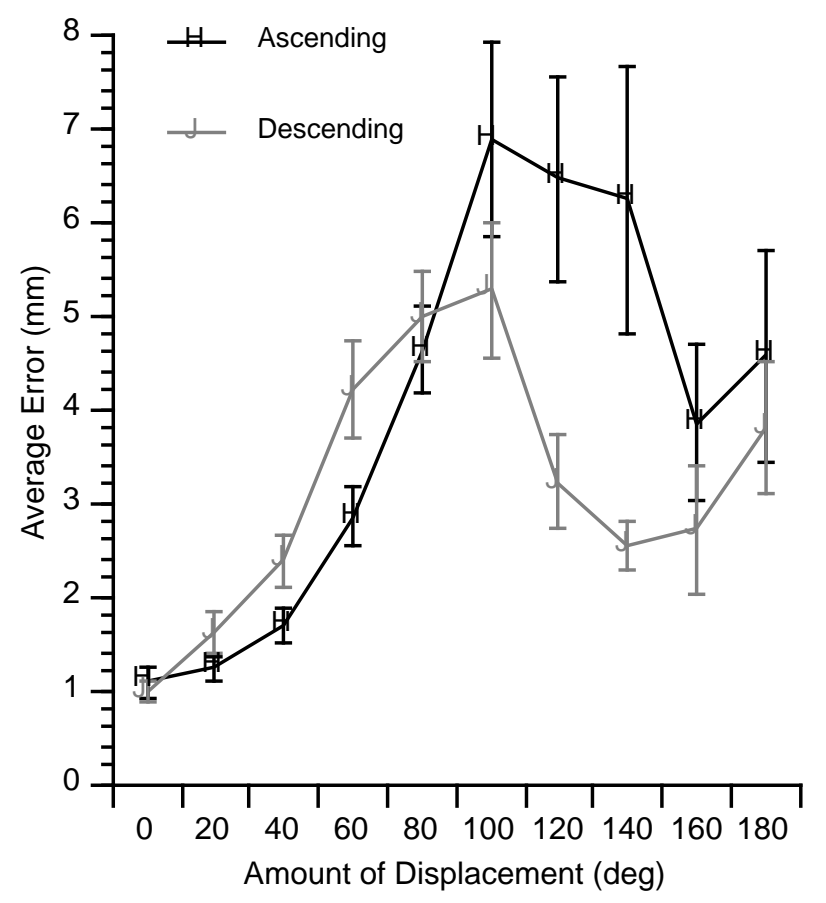

Figure 3. Performance error plotted as a function of both order and level of coordination displacement.

Alternatively, descending performance was superior for the $120^{\circ}$ and $140^{\circ}$ displacements $(t(31)=-$ 2.98, $p<.006 ; t(31)=-2.80, p<.009)$. However, no statistically reliable performance differences were observed for the $160^{\circ}$ or $180^{\circ}$ displacements. The $180^{\circ}$ displacement, in contrast to the $80^{\circ}$ and $100^{\circ}$ displacements, is a behaviorally simple transformation. One moves the cursor opposite the direction of the intended target. This displacement is viewed as a more difficult analog of the $0^{\circ}$ displacement. Although the evidence for the hysteresis loop is weaker between the $180^{\circ}$ and $100^{\circ}$ displacements, probably due to the performance variability mentioned above, qualitatively the pattern is similar to that observed for the displacements that were less that $100^{\circ}$.

These results are consistent with the hysteresis pattern and display performance symmetry. Beginning with the ascending condition, performance error was conserved as the displacements incremented away from the normal coordination pattern until the $80^{\circ}$ and $100^{\circ}$ displacements were encountered. As the displacements continued to increase, performance was slow to recover, despite the fact that the displacements were becoming easier to perform.

The performance pattern for the descending displacements was similar. The transformation from $0^{\circ}$ to $180^{\circ}$ was relatively simple (compared to adjacent displacements; see [6]). As the participants incremented away from this mapping, performance was preserved relative to ascending performance. Again, a local maximum was encountered with the $100^{\circ}$ and $80^{\circ}$ displacements; likewise, performance was also slow to recover with respect to ascending performance under the same displacements.

\section{Discussion}

Overall, the results of this experiment were consistent with a pattern of hysteresis. On average (i.e., collapsing across the order variable), the 80 and 100 degree displacements produced maximum error. The participants performance was conserved in cases where they approached these difficult displacements from less disruptive displacements. One way to view these results is that the hysteresis pattern observed across the 180 to 100 degree displacements is a replication of the hysteresis pattern that was observed for the 0 to 80 degree displacements. Thus, better performance was not simply associated with a particular presentation order of displacements; it was contingent on the direction of ordered presentation irrespective of the absolute point of reference.

Although the practice counterbalancing was designed to bias the experiment against the observed hysteresis pattern, fatigue effects could produce a pattern of results that are consistent with these data. However, there are reasons to suggest that the role of fatigue was small. Each participant spent less than 15 minutes to complete the entire experimental session, this included time used to receive instructions and be debriefed. In addition, another group of participants employed the circle task by first completing five practice 0 degree 
displacements followed by ten trials of the difficult 90 degree displacement. In each of these cases performance was better in the final 90 degree trials than in the initial 90 degree trials.

From the standpoint of actual surgical practice, these results suggest ways to help a surgeon, for example, to overcome coordination disorientation during a procedure. A surgeon could, within the constraints of the particular operational situation, draw their coordination pattern closer to a normal or familiar orientation and then increment back to the pattern that is required to complete the specific task of interest. This behavioral strategy could be especially important for beginning surgical residents, who become disoriented rather easily. In situations where the resident has difficulty recovering from a displacement disorientation, the attending surgeon usually takes over control of the surgery, precluding further hands on training. This tends to limit the amount of time and practice that the resident receives. The suggested strategy could help break this negative feedback cycle, where poor performance results in fewer opportunities for practice.

In addition, these results suggest how we may understand variability in the dynamics of hand-eye coordination. Hysteresis is a topological pattern that is interpreted to suggest multistability [24]. Apparently, an adequate description of hand-eye coordination must include information concerning prior system manipulations in addition to the current levels of manipulated variables. In other words, contextual information becomes central to the description because the coordination system seems to leverage context to produce behavior.

\section{Conclusion}

The hysteresis patterns observed here parallel in quality the hysteresis behavior of rather simple nonlinear dynamic systems. Hysteresis underscores the role of the contextual constraints in both an understanding and control of behavior. It also motivates dynamic systems theory as a general framework for understanding coordination [4, 30, 31, 33, 39]. My future research will employ additional empirical and analytical tools from dynamic systems theory toward a general understanding of hand-eye coordination.

\section{Acknowledgments}

I gratefully acknowledge the people who participated in the development of this research program; Guy Van Orden provided helpful suggestions and comments. John M. Flach helped with the early stages of this project. The experimental software was developed by Kenn J. Angell. Assistance with the management and analysis of the experimental data was provided by Frank Arias. This research was supported in part by the Department of Psychology at Arizona State University.

\section{References}

[1] Abraham, R. A., \& Shaw, C. D. (1992). Dynamics: The geometry of behavior. Redwood City, CA: Addison-Wesley.

[2] Bedford, F. L. (1989). Constraints on learning new mappings between perceptual dimensions. Journal of experimental psychology: Human perception and performance, 15(2), 232-248.

[3] Bedford, F. L. (1993). Perceptual and cognitive spatial learning. Journal of experimental psychology: Human perception and performance, 19(3), 517530.

[4] Bernstein, N. (1967). The Co-ordination and regulation of movements. New York: Pergamon.

[5] Brokate, M., \& Sprekels, J. (1996). Hysteresis and phase transitions. New York: Springer-Verlag.

[6] Cunningham, H. A. (1989). Aiming error under transformed spatial mappings suggests a structure for visual-motor mappings. Journal of experimental psychology: Human perception and performance, 15(3), 493-506.

[7] Cunningham, H. A., \& Pavel, M. (1993). Target axis effects under transformed visual-motor mappings. In Ellis, S. R. (Ed.) Pictorial communication in virtual and real environments. Washington, DC: Taylor \& Francis.

[8] Cunningham, H. A., \& Welch, R. B. (1994). Multiple concurrent visual-motor mappings: Implications for models of adaptation. Journal of experimental psychology: Human perception and performance, 20(5), 987-999.

[9] Cuschieri, A. (1995). Wither minimal access surgery: Tribulations and expectations. American Journal of surgery, 169, 9-19.

[10] Dominguez, C. O., Flach, J. M., McKellar, D. P., \& Dunn, M. (1996). Using videotaped cases to elicit perceptual expertise in laparoscopic surgery. Proceedings of the 3rd Annual Symposium on Human Interaction with Complex Systems, 116123.

[11] Flook, J. P., \& McGonigle, B. O. (1977). Serial adaptation to conflicting prismatic rearrangement effects in monkey and man. Perception, 6, 15-29.

[12] Gibson, J. J. (1966). The senses considered as perceptual systems. Boston: Houghton Mifflin.

[13] Gibson, J. J. (1979). An ecological approach to visual perception. Boston: Houghton Mifflin.

[14] Hatlie, M. J. (1993). Climbing the 'learning curve': New technologies, emerging obligations. Journal of the American Medical Association, 270(11), 
1364-1365.

[15] Held, R., \& Durlach, N. (1993). Telepresence, time delay and adaptation. In Ellis, S. R. (Ed.) Pictorial communication in virtual and real environments. Washington, DC: Taylor \& Francis.

[16] Helmholtz, H. V. (1866/1962). Treatise on physiological optics, Vol. 3. New York: Optical society of America.

[17] Holden, J. G. (1996). Perceptual-motor coordination in an endoscopic surgery simulation. Unpublished master's thesis, Wright State University. Dayton, $\mathrm{OH}$.

[18] Holden, J. G., \& Flach, J. M. (1996). Hand-eye coordination in an endoscopic surgery simulation. Proceedings of the 3rd Annual Symposium on Human Interaction with Complex Systems, 110115.

[19] Howard, I. P. (1982). Adaptation to discordant stimulation. In Howard, I. P. (Ed.). Human visual orientation. New York: John Wiely \& Sons.

[20] Kelso, J. A. S., Ding, M., \& Schoner G. (1993). Dynamic pattern formation: A primer. In Smith, L. B., \& Thelen, E. (Eds.) A dynamic systems approach to development. Cambridge, MA: MIT press.

[21] Kim, W. S., Tendick, F., \& Stark, L. (1993). Visual enhancements in pick-and-place tasks: Human operators controlling a simulated cylindrical manipulator. In Ellis, S. R. (Ed.) Pictorial communication in virtual and real environments. Washington, DC: Taylor \& Francis.

[22] Michaels, C. F., \& Carello, C. (1981). Direct perception. New Jersey: Prentice-Hall.

[23] McGonigle, B. O., \& Flook, J. (1978). Long-term retention of single and multistate prismatic adaptation by humans. Nature, Vol. 272, 364-366.

[24] Poston, T., \& Stewart, I. (1978). Nonlinear modeling of multistable perception. Behavioral Science, Vol. 23, 318-334.

[25] Robinson, D. A. (1972). Eye movements evoked by collicular stimulation in the alert monkey. Vision research, 12, 1795-1808.

[26] Stewart, I. N., \& Peregoy, P. L. (1983). Catastrophe theory modeling in psychology. Psychological bulletin, Vol. 94(2), 336-362.
[27] Stratton, G. M. (1896). Some preliminary experiments on vision without inversion of the retinal image. Psychological review, 3, 611-617.

[28] Stratton, G. M. (1897). Vision without inversion of the retinal image. Psychological review, 4(4), 341-360.

[29] Tendick, F., Jennings, R.W., Tharp, G., \& Stark, L. (1993). Sensing and manipulation problems in endoscopic surgery: Experiment, analysis, and observation. Presence, 2(1), 66-81.

[30] Thelen, E. (1995). Motor development: A new synthesis. American psychologist, 50, (2), 79-95.

[31] Thelen, E., \& Smith, L. B. (1994). A dynamic systems approach to the development of cognition and action. Cambridge, MA: MIT press.

[32] Tuller, B., Case, P., Ding, M., \& Kelso, J. A. S. (1994). The nonlinear dynamics of speech categorization. Journal of experimental psychology: Human perception and performance, Vol. 20, (1), 3-16.

[33] Turvey, M. T., \& Carello, C. (1986). The ecological approach to perceiving-acting: A pictorial essay. Acta Psychologica, 63, 133-155.

[34] Varela, F. J., Thompson, E., \& Rosch, E. (1991). The embodied mind. Cambridge, MA: MIT press.

[35] Visintin, A. (1994). Differential models of hysteresis. New York: Springer-Verlag.

[36] Welch, R. B. (1978). Perceptual modification: Adaptation to altered sensory environments. New York: Academic press.

[37] Welch, R. B. (1986). Adaptation of space perception. In K. R. Boff, L. Kaufman, \& J. P. Thomas (Eds.). Handbook of perception and human performanceVolume I, Sensory processes and perception. New York: Wiley \& sons.

[38] Welch, R. B. (1993). Adapting to variable prismatic displacment. In Ellis, S. R. (Ed.) Pictorial communication in virtual and real environments. Washington, DC: Taylor \& Francis.

[39] Zanone, P. G., \& Kelso, J. A. S. (1991). Experimental studies of behavioral attractors and their evolution with learning. In J. Requin, \& G. E. Stelmach (Eds.), Tutorials in motor neuroscience, 121-133. Nederlands: Kluwer. 